\title{
An Electronic Medical Record in Pediatric Medical Education: Survey of Medical Students' Expectations and Experiences
}

\author{
Daryl R. Cheng ${ }^{1,2,3}$ Thomas Scodellaro ${ }^{1}$ Wonie Uahwatanasakul ${ }^{2,3}$ Mike South ${ }^{1,2,3}$
}

\footnotetext{
${ }^{1}$ EMR Team, The Royal Children's Hospital Melbourne, Parkville, Australia

${ }^{2}$ Department of General Medicine, The Royal Children's Hospital Melbourne, Parkville, Australia

${ }^{3}$ Department of Paediatrics, Faculty of Medicine, Dentistry and Health Sciences, University of Melbourne, Carlton, Australia
}

Appl Clin Inform 2018;9:809-816.

\author{
Address for correspondence Daryl R. Cheng, MBBS, MPH, FRACP, \\ Department of General Medicine, The Royal Children's Hospital \\ Melbourne, 50 Flemington Road, Parkville, VIC 3052, Australia \\ (e-mail: daryl.cheng@rch.org.au).
}

\section{Abstract}

Keywords

- EHR

- medical student

- education

- curriculum
Objective This study sought to quantitatively characterize medical students' expectations and experiences of an electronic health record (EHR) system in a hospital setting, and to examine perceived and actual impacts on learning.

Methods Medical students from July to December 2016 at a tertiary pediatric institution completed pre- and postrotation surveys evaluating their expectations and experience of using an EHR during a pediatric medicine rotation. Survey data included past technology experience, EHR accessibility, use of learning resources, and effect on learning outcomes and patient-clinician communication.

Results Students generally reported high computer self-efficacy $(4.16 \pm 0.752$, mean \pm standard deviation), were comfortable with learning new software $(4.08 \pm 0.771)$, and expected the EHR to enhance their overall learning $(4.074 \pm 0.722)$. Students anticipated the EHR to be easy to learn, use, and operate, which was consistent with their experience (pre 3.86 vs. post $3.90, p=0.56$ ). Students did not expect nor experience that the EHR reduced their interaction, visual contact, or ability to build rapport with patients. The EHR did not meet expectations to facilitate learning around medication prescribing, placing orders, and utilizing online resources. Students found that the EHR marginally improved feedback surrounding clinical contributions to patient care from clinicians, although not to the expected levels (pre 3.50 vs. post 3.17, $p<0.01$ ).

Conclusion Medical students readily engaged with the EHR, recognized several advantages in clinical practice, and did not consider their ability to interact with patients was impaired. There was widespread consensus that the EHR enhanced their learning and clinician's feedback, but not to the degree they had expected.

\section{Background and Significance}

The transition within health care institutions from paperbased charting to electronic health record (EHR) systems

received

April 18, 2018

accepted after revision

September 18, 2018 unlocks enormous potential to enhance medical student education. The increasing availability of integrated EHRs has led to support from both educational (c) 2018 Georg Thieme Verlag KG Stuttgart · New York
DOI https://doi.org/ 10.1055/s-0038-1675371. ISSN 1869-0327. 
and clinical organizations for its use to enhance teaching and learning.

Existing literature demonstrates learning benefits when medical education initiatives utilize integrated EHRs in developing patient care abilities, communication skills, and systems-based thinking. ${ }^{1-3}$ In particular, medical education outcomes specifically from EHR usage are being more widely evaluated. ${ }^{4-6}$ This research stems mainly from a North American context-where EHR uptake has been the most prevalent and therefore usage within medical education is common. ${ }^{7}$

Current practice guidelines from clinical and academic bodies alike strongly encourage the involvement of medical student interaction within EHRs. This includes using note templates and other adjuncts, as well as the provision of opportunities to practice computerized test or medication order entry and observe the use of decision aid systems. ${ }^{7,8}$ Other tools such as interactive e-learning modules and high fidelity simulated and virtual patient or clinical scenarios can also be used to enhance learning. However, among established EHRs there remains considerable interinstitution and interdepartment variability in medical student access and usage policies; sometimes even with disparate views between students and educators. ${ }^{9-11}$ This is despite indications that preventing medical students from contributing to patients' records due to administrative or billing policies is more detrimental than beneficial. ${ }^{12}$

Any potential educational outcomes are also affected by the learning environment. Within pediatric medicine, there are unique nuances such as weight-based dosing, immunizations, newborn screening, and developmental milestones which currently do not feature in all EHRs. ${ }^{13}$ In order for learners to appropriately and adequately grasp these important concepts, EHRs must include workflows designed to integrate and demonstrate these functionalities. ${ }^{14,15}$

The extent to which medical students readily engage with EHR systems is yet to be demonstrated. Current medical students are often excitingly perceived as more "technologysavvy" and therefore receptive to new technologies compared with more senior clinicians, suggesting they may readily engage in clinical education via an integrated EHR. $^{16,17}$ Unfortunately, there is a paucity of data directly evaluating medical students' beliefs about EHRs and their associated implications to aid in ongoing strategy and curricula development. ${ }^{18}$
Nonetheless, any potential educational benefits are juxtaposed against perceived inefficiencies or detrimental effects. Previous studies indicate EHRs may unwittingly undermine learning by "thinking for the student," compromising patient face-to-face contact and excluding students from clinical tasks. ${ }^{19,20}$ A summary of these benefits and disadvantages is outlined in - Table 1 .

\section{Objective}

It is within this context that this study seeks to quantitatively characterize and measure medical students' expectations, interactions with, and experiences of an integrated EHR system on their pediatric education experience via a questionnaire survey. Furthermore, this study seeks to assess the perceived impact of such a system on student-patient interactions and learning objectives.

\section{Methods}

\section{Setting and Participants}

Our tertiary academic teaching hospital, The Royal Children's Hospital Melbourne ( $\mathrm{RCH})$, has a partnership with The University of Melbourne (UoM), and provides pediatric clinical rotations and learning opportunities for its medical students. The hospital's recent certification as a Healthcare Information and Management Systems Society Level 6 hospital with an integrated EHR system (Epic EHR, Wisconsin, United States) implemented in April 2016 provided a prime opportunity to integrate and evaluate the use of its comprehensive integrated EHR for medical student education.

Prior to the implementation of an integrated EHR, a "bestof-breed" technology system existed. Handwritten notes, referrals, medication charts, and orders were combined with an electronic laboratory review system and a scanned read-only medical record viewer.

A prospective cohort study was conducted from July to December 2016 comprising all medical students in the academic year that utilized the EHR as part of their learning experience-after the EHR implementation on April 30, 2016. Students on peripheral hospital placements outside $\mathrm{RCH}$ or elective medical students not from the UoM were excluded from the study. Students were not involved in the conversion or cutover process to the integrated EHR; their first

Table 1 Benefits and disadvantages of an EHR in medical student learning

\begin{tabular}{|l|l|}
\hline Potential Benefits $^{1}$ & Potential Disadvantages $^{20,22}$ \\
\hline - Improved and streamlined workflow by reducing time & - Failure to effectively teach synthesis of clinical \\
needed to search for information and retrieve data & information/critical thinking skills as students become \\
- Enhanced self-directed learning process by facilitating & reliant on order sets and decision support \\
best practice guideline and resource integration & - Failure to develop effective communication skills \\
- Enhanced communication between students and & between students, healthcare teams and patients \\
patients if optimized correctly & - Students may develop poor documentation skills by \\
- Allowing for direct feedback between students and & simply copying and pasting information or previous \\
supervisors & notes. \\
- Ability to track task completion or assessment easily & Potential to exclude students from clinical tasks which \\
& are important in holistic learning \\
\hline
\end{tabular}


interaction with the EHR was at a mandatory training session as outlined below.

Within the RCH EHR, medical students were given full access to patient clinical information and were able to interact with the system by entering clinical notes, reviewing data, and placing limited orders within the system in both inpatient and outpatient settings. Student notes at our institution form part of the medical record, and are attested by residents or staff pediatricians. Two medical student note templates (admission and ward round note) were designed for student use; otherwise there were no specific workflows, order sets, or training materials or modules for students. Supervising clinician review and cosign for all student entries or orders within the EHR was and continues to be mandatory.

All students were provided with a standardized 4-hour EHR training session prior to commencement of their placement, where the functionalities and opportunities to participate in clinical care and learning using the EHR were demonstrated and practiced. The optional opportunity to further experiment within a training environment at their leisure was also provided.

After the training session, students were invited in person to complete a paper-based prelive EHR use survey 1 week prior to commencing their pediatric rotation in which they evaluated their expectations of the EHR and its impacts on their education. This was followed by a reminder email 48 hours later to complete and return any outstanding surveys. Students then completed a corresponding postcommencement survey immediately after concluding their rotation which assessed their experience at the end of their rotation. There were no monetary or other incentives to participate in the study.

Ethics approval was granted for the project by RCH Human Research Ethics Committee (39196A).

\section{Questionnaire/Measurement Tool}

An anonymous 25-item pre- and post-EHR use multiplechoice and free-text questionnaire was used for this study (see - Supplementary Material, available in the online version). It was adapted and expanded from previous surveys assessing the impact and perceptions of EHRs on medical student learning in the North American setting. ${ }^{18,19}$

The survey evaluated five main areas-past technology experience and proficiency, the accessibility of the EHR, use of learning resources, impact on learning outcomes, and impact on patient-clinician communication. For this study, the latter three were considered dependent variables. Previous evidence has shown that the perceived ease of use and effect on learning are strong predictors for physician acceptance and use of technology tools within their workplace. ${ }^{18}$ Responses were quantified using a five-point Likert scale from "Strongly Disagree" to "Strongly Agree," and are reported as mean \pm standard deviation (SD).

\section{Statistical Consideration}

Deidentified data were stored and analyzed using Microsoft Excel (Redmond, Washington, United States) and Stata (Sta- tacorp, Texas, United States). Mann-Whitney U-testing was used in the study's primary analysis to assess for differences in mean and median results in the pre- and postintervention surveys (i.e., perceived and actual experience).

A multivariate analysis was then conducted assessing the relationship between student factors (age, gender, history of EHR use, and assessed computer proficiency) and each of the dependent variables while controlling for other independent variables. Statistical significance was reported as $p<0.05$.

\section{Results}

Of 108 eligible students, there were 94 (response rate, 87.0\%) and $87(80.6 \%)$ responses to the pre- and postcommencement surveys, respectively. There was a slight male majority (56.6\%), with a median age of 24 years (interquartile range, 23-26).

The majority of students (67.7\%) reported previous experience with non- or partially integrated EHR systems, predominantly in community-based settings (e.g., family medicine practice). They generally reported high computer self-efficacy (Likert score, $4.16 \pm 0.752$, mean \pm SD) and were comfortable with learning new software $(4.08 \pm 0.771)$.

\section{Ease of Use}

Students anticipated the EHR to be easy to learn, use, and operate, which was consistent with their actual experience ( - Fig. 1). Students expected they would prefer to use an EHR over paper-based systems to search for (pre 4.19 vs. post $4.12, p=0.54$ ), review (pre 4.22 vs. post $4.39, p=0.16$ ), and follow-up (pre 4.38 vs. post $4.39, p=0.94$ ) patient information; this was again reinforced after actual experience using the system ( - Fig. 1). Free-text responses from students indicated that they felt more comfortable with the EHR compared with the paper system, and also increased their frequency of reviewing patient information-often in their own time outside formal clinical settings.

\section{Learning Outcomes}

The EHR enabled student perceptions that their clinical documentation was more complete than expected (pre 3.82 vs. post $4.09, p=0.02$ ). Students felt that the EHR marginally improved feedback surrounding clinical contributions to patient care from clinicians, although not to the preuse expected levels (pre 3.50 vs. post 3.17, $p<0.01$ ).

Likewise, the EHR's integrative tools did not meet student expectations to better facilitate learning in specific areasincluding placing orders (pre 3.90 vs. post $3.40, p<0.01$ ), medication interactions (pre 3.85 vs. post $3.16, p<0.001$ ), and accessing online medication resources (pre 3.85 vs. post 3.48, $p<0.01$ ) (-Fig. 2).

\section{Communication}

Students did not expect nor experience that the EHR would reduce their perceived interaction or ability to build rapport with patients. There was a trend toward reduced visual 


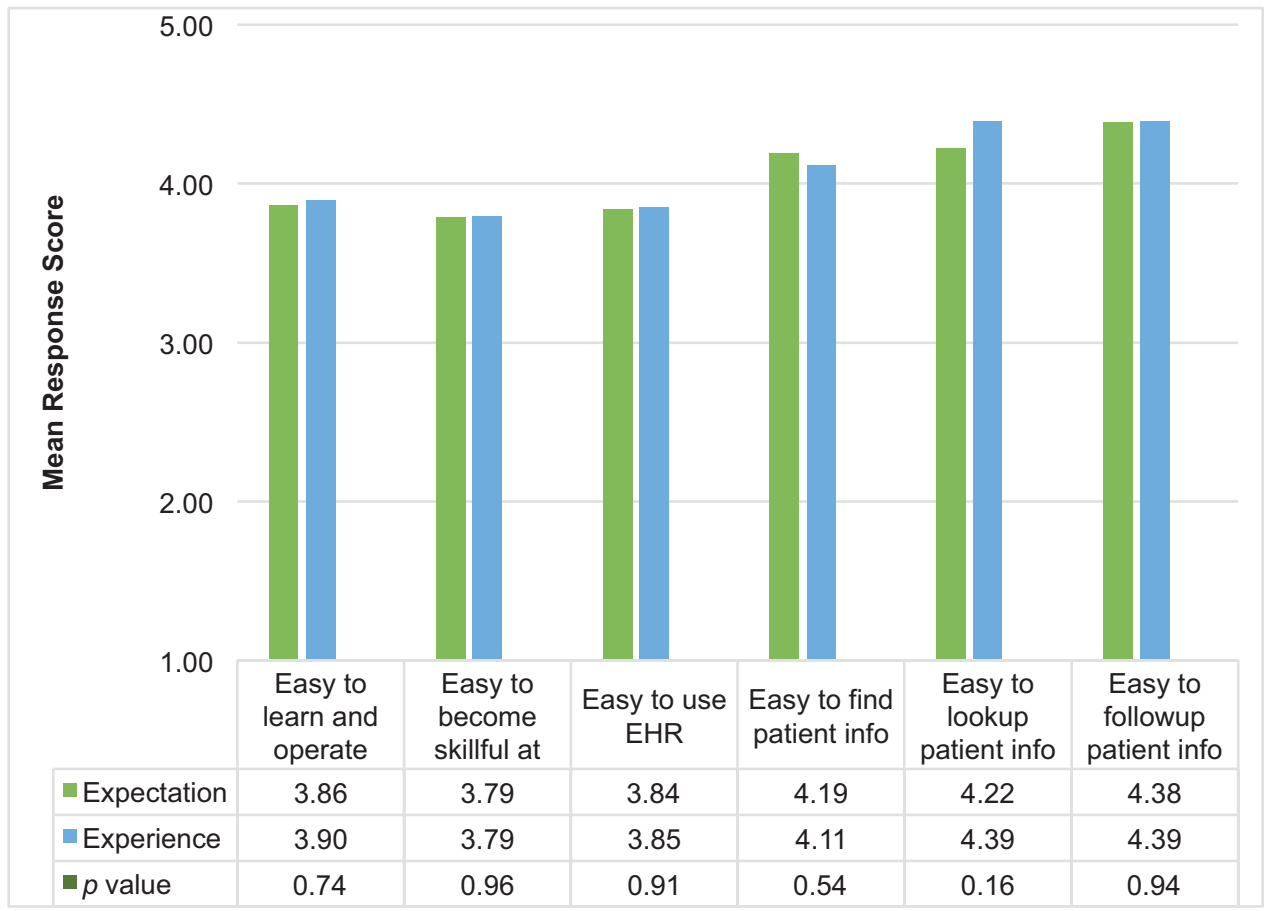

Fig. 1 Mean response scores for electronic health record (EHR) and impact on ease of use.

contact between students and patients, although students indicated at worst this was no different than preintegrated EHR practice (pre 2.60 vs. post $2.92, p=0.03$ ). Overall, students both perceived and felt that the EHR enhanced their ability to contribute to the clinical team (pre 4.14 vs. post $4.20, p=0.31$ ) ( Fig. 3).

\section{Multivariate Analysis}

Multivariate analysis showed that student factors explained more of the variance in perceived usability $\left(R^{2}=0.25\right)$ than resource and learning $\left(R^{2}=0.05\right)$ or communication $\left(R^{2}\right.$ $=0.04$ ) improvements. Nonetheless, there remains a significant degree of unexplained variance.

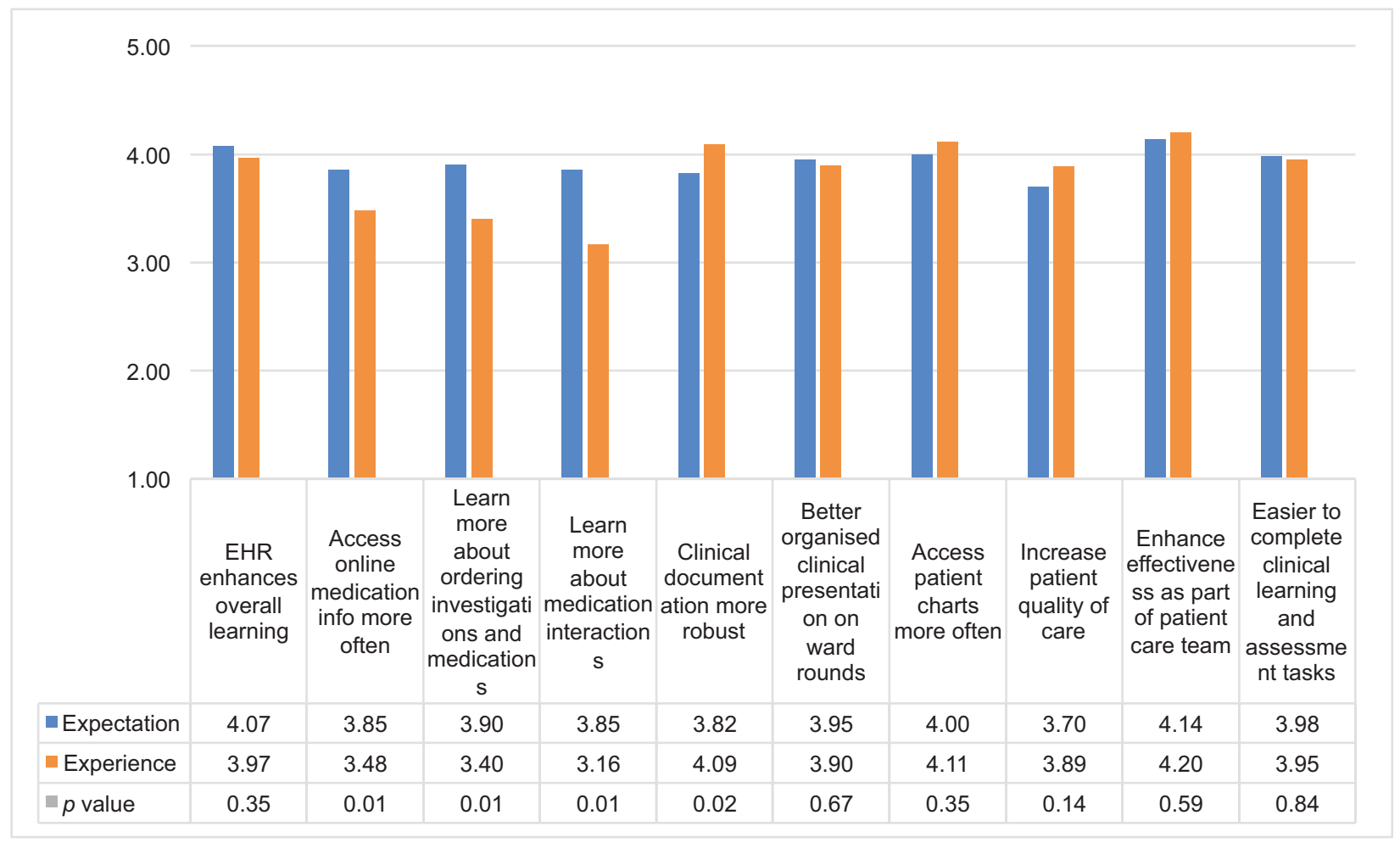

Fig. 2 Mean response scores for electronic health record (EHR) and impact on learning outcomes. 


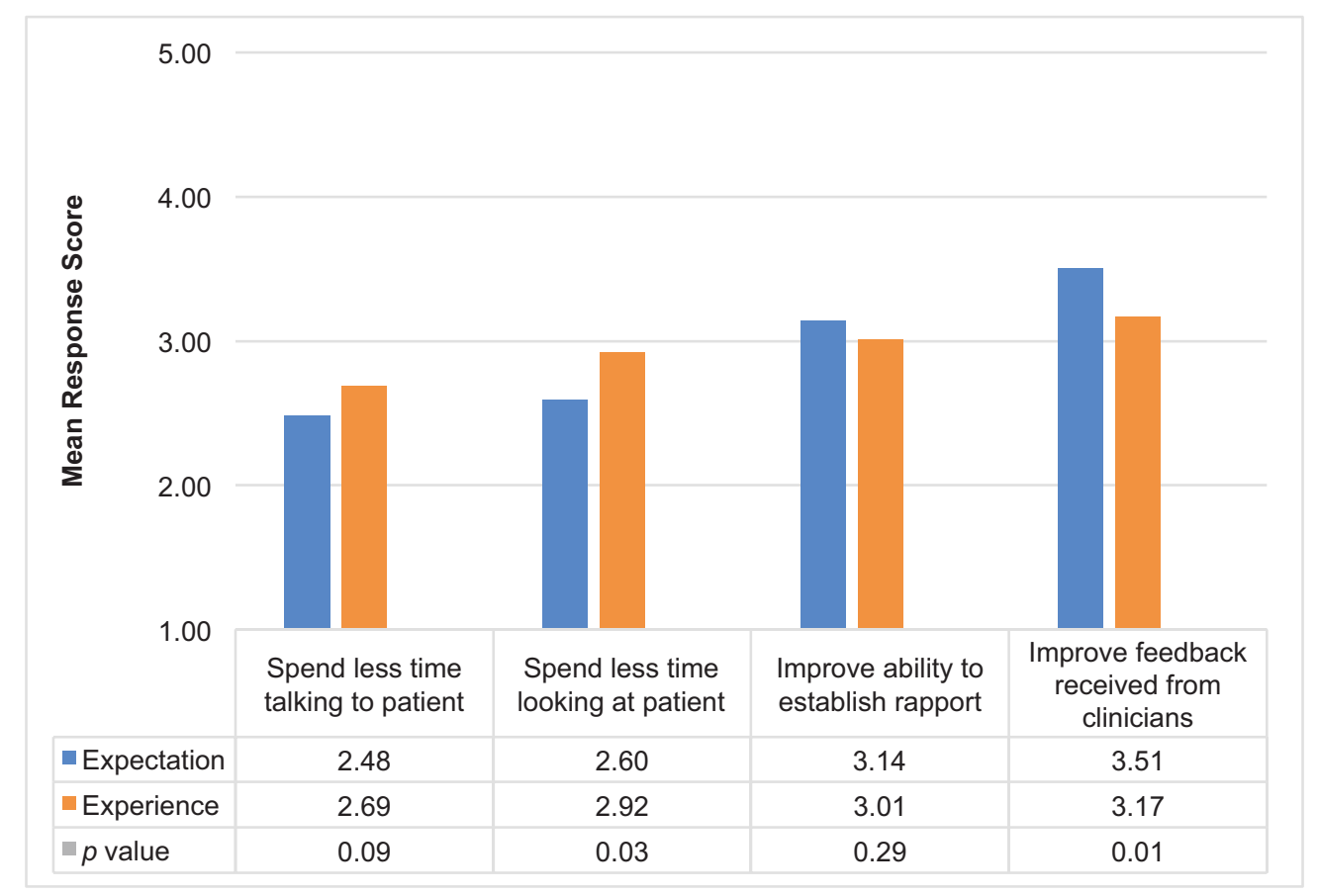

Fig. 3 Mean response scores for electronic health record (EHR) and impact on communication.

When controlling for other variables, a higher proficiency score was associated with higher perceived ease of use $(p<0.001)$, ease and depth of learning $(p<0.001)$, but not enhanced communication with patients when using the EHR instead of paper charts. Age, gender, and a history of EHR use were not significantly associated with perceived ease of use, learning, or communication.

\section{Discussion}

To our knowledge, this is the first study in an Australasian context evaluating both the expectations, interactions with, and learning experiences of medical students utilizing an integrated EHR as part of their educational experience. It demonstrates clear student interaction, usage, and uptake of an integrated EHR, as well as perceived benefits for medical student education in various domains across primarily inpatient but also ambulatory settings.

Not surprisingly, medical students were keen to utilize the EHR as part of their learning experience. Given that most student-EHR interactions were self-directed, this not only supports intergenerational perceptions of younger people's willingness to engage in new technologies but validates our EHR framework designed to maximize student participation without compromising patient safety. Even among individuals reluctant to engage with the EHR, one would still expect "osmotic" learning to occur from the sheer amount of time spent at computer workstations as part of a medical team. ${ }^{4}$

There was a clear association between students who had a higher technical proficiency and greater EHR ease of use and depth of learning; the self-directed exploration learning methods may have been most beneficial for these students.
Indeed, Lehmann et al have reported similar findings in surveys of outpatient-based pediatricians where positive factors such as younger age and higher technical proficiency reinforce adoption and uptake of EHRs. ${ }^{21}$

Overall, the majority of students recognized and expected the EHR to convey several advantages to both their clinical practice and learning experience. With respect to core EHR functions, students' perceived experience often matched or bettered their expectations. This included students' ability to review information and also facilitate electronic communication between providers. The system's effect on learning through ordering functions, however, failed to meet student expectations. It is, however, important to consider that core functionality does not necessarily translate to an enhanced educational experience, given many administrative tasks facilitated by the EHR each carry variable educational benefit.

Nonetheless, this highlights that education also deserves consideration as a key outcome when institutions are looking to adopt or expand their EHR. The priorities of improving efficiency and patient safety via an EHR should be balanced against the need to ensure enhanced educational experiences and best learning outcomes for students.

\section{User Experience}

Students' interaction and user experience within the EHR was overwhelmingly positive. They not only found the EHR easy to learn, operate, and become skillful at, but preferred to review patient information electronically compared with paper charts. While an element of this could be attributed to students' paucity of experience with an electronic system of this nature, and therefore underestimates ease of usability of the system, it likely reflects the ability of current medical 
students to embrace technology for enhancement of their learning. This is particularly telling, given it demonstrates the accessibility and availability of information to be not only key factors in maintaining quality and safe patient care, but are core priorities among the current generation of medical learners.

This positive user experience referred to in student comments enabled students to have up-to-date patient clinical information, but also underpinned all other aspects of EHR interactions, patient care, and learning. Indeed, one would expect a positive student experience to enable a smoother transition for individuals becoming a junior doctor-who will in turn be the primary users of EHRs for everyday inpatient care. $^{22}$ It is interesting to note, however, that technical proficiency rather than history of EHR use is associated with ease of use. This suggests that learning methods should not necessarily only focus on specific EHR functions but could also allow time for the development of overall computer skills for long-term benefit.

\section{Learning Experience}

Students reported a significant improvement in the perceived completeness of clinical documentation compared with paper note documentation. This has been well described in previous literature, and often forms the backbone of medical student's contributions within an integrated EHR. ${ }^{19}$ Students were able to leverage on real-time access to patient information, the availability of note templates and shortcut tools, and the nontime limited ability to consider and compose their note, and to have it electronically checked by supervising doctors who would then cosign their work.

Despite increased documentation, feedback to students from clinicians was seen to be marginally improved, but not to the expected levels. This may have been secondary to clinicians also familiarizing themselves with a new systemthus resulting in limited time to provide feedback. It may also be that EHR workflows themselves are a barrier to feedbackin that students are able to contribute directly to the patient EHR, but remote annotation and cosigning of notes or orders by supervising clinicians meant that the feedback may not have been communicated directly to the student as much or as promptly as they had expected.

Tools incorporated within the EHR to facilitate learning around medication prescribing and computerized order entry did not enhance student learning to their preusage expectations. However, students still felt they assisted learning more than pre-EHR methods. This discrepancy between experience and expectations, especially in the area of ordering investigations and medications, is likely due to adjunct learning resources such as instructional videos and EHR training packages being targeted at a junior doctor level, resulting in less tangible benefits for students. It may also reflect student unfamiliarity with concepts of orders and prescribing in a pediatric setting, especially in regards to weight-based prescribing-with students overestimating the utility of the EHR to aid learning in these areas. ${ }^{23}$ Furthermore, individual EHR deficiencies in medication ordering and interactions may have also contributed to the discrepancy.

This highlights that EHRs could be better at facilitating learning around specific nuances in clinical practice. If their functionality remains incomplete, this could not only result in poor user experience and frustration, but also potentiate harm through misuse or nonadoption as a learning tool. ${ }^{13,14}$

While workflow and efficiency gains within the EHR may be beneficial, they may also unwittingly reduce the need for students to self-search as part of their learning. This is becoming more relevant, as learners increasingly have no prior interactions with paper-based medical record documentation. Thus, if learners rely on EHRs as their sole learning platform, they risk forfeiting their discovery process and diminishing development of critical thinking skills. ${ }^{24,25}$

\section{Communication Experience}

Reassuringly, transition to an integrated EHR did not diminish student perceived interaction or rapport with patients. While this is a phenomenon that has been observed previously in some settings, ${ }^{19}$ Alkureishi et al have shown no negative impact on either patient satisfaction or patientdoctor communication. ${ }^{26}$ Nonetheless, it remains a challenge that clinical teams and learners must guard against.

The vast majority of students felt that the EHR improved students' ability to contribute to the clinical team. Although this contribution may have taken the form of assistance in administrative tasks such as completing discharge summaries, one can postulate that it is just as important for students to have EHR facilitate administrative as well as clinical learning. This serves as a preparatory step for a smoother transition to expanded EHR use as a future medical practitioner.

\section{Limitations}

The scope of this study meant learning experiences were based on a cohort study of students and their expectations and experiences. This approach was chosen due to perceived variability in past student experience with EHRs. Hence by assessing students' expectations, an established baseline could then be used to assess students' experiences. While the survey tool used in this study was adapted from existing surveys in the literature, it has not been validated in this specific subpopulation. Thus, care needs to be taken when drawing and generalizing conclusions. Future studies using validated tools to assess learning enrichment will build on this study's findings.

All students received a standardized EHR training session prior to commencement, as per mandatory requirement for all institutional staff or students. While this may have impacted students' perceptions and perspectives on the EHR, this was minimized by standardizing training content and delivery with focus on workflows rather than ways to optimize learning or education. ${ }^{27}$

This study was based at a pediatric hospital with specific workflows and systems; thus, findings may not apply to all medical and/or adult rotations. The EHR evaluated within our study is currently the only installation of its kind in Australasia, 
and thus may differ from other installations of the same software. However, many of the discussion points raised here are generalizable to EHRs and medical learning experiences, and thus may prove useful across various clinical settings.

\section{Conclusion}

Medical students readily engaged with an integrated EHR, recognizing several advantages in their learning experience across various domains. Students did not consider their ability to interact with patients to be impaired by using the EHR. There was widespread consensus that the EHR enhanced their learning and clinician's feedback, but not to the degree they had expected.

Overall, an integrated EHR is both a desired and beneficial learning tool for medical students completing a pediatric rotation. Future studies looking specifically at certain domains and subgroups will add to current evidence.

\section{Clinical Relevance Statement}

The priorities of improving efficiency and patient safety via an EHR should be balanced against the need to ensure enhanced educational experiences and best learning outcomes for students. Given the multifactorial nature of utilizing the EHR effectively, input from a variety of stakeholders is crucial for success. Medical education deserves consideration as a key outcome when institutions are looking to adopt or expand their EHR.

\section{Multiple Choice Questions}

1. Which of the following is true regarding EHRs and impact on ordering and prescribing?

a. They enhance medical student learning beyond preEHR expectations.

b. Simplifying tasks may reduce learner development of critical thinking.

c. Learners feel electronic ordering does not enhance learning more than paper-based methods.

d. Learners were more likely to access medication prescribing resources after using the her.

Correct Answer: The correct answer is option b. By automating tasks or at the very least reducing the number of steps required to order a medication, time and efficiency savings are gained. However, the critical thinking skills that learners develop through this process-particularly individual patient critical analysis may be jeopardized. This in turn may impact the risk of medication and order prescribing errors-an unwanted effect that is within the future research scope.

2. Which of the following is the most likely contributing factor to potential nonadoption of EHR as a learning tool?

a. Diminished critical thinking skills.

b. Standardized user training.

c. Poor EHR functionality and design.

d. Effective clinical decision support aids.
Correct Answer: The correct answer is option c. Poor EHR functionality and design, especially if it does not address specific nuances (e.g., pediatric weight-based dosing), is a key factor in user nonadoption for learning. Diminished critical thinking skill is a result of overreliance on EHR for learning. Effective clinical decision support tools and standardized user training aid in enhancing the EHR for educational and functionality purposes.

\section{Protection of Human and Animal Subjects}

This study was performed in compliance with the World Medical Association Declaration of Helsinki on Ethical Principles for Medical Research Involving Human Subjects and was reviewed by RCH Human Research Ethics Committee (39196A).

\section{Conflict of Interest}

None.

\section{References}

1 Tierney MJ, Pageler NM, Kahana M, Pantaleoni JL, Longhurst CA. Medical education in the electronic medical record (EMR) era: benefits, challenges, and future directions. Acad Med 2013;88 (06):748-752

2 Stephens MB, Gimbel RW, Pangaro L. Commentary: the RIME/EMR scheme: an educational approach to clinical documentation in electronic medical records. Acad Med 2011;86 (01):11-14

3 Keenan CR, Nguyen HH, Srinivasan M. Electronic medical records and their impact on resident and medical student education. Acad Psychiatry 2006;30(06):522-527

4 Chi J, Kugler J, Chu IM, et al. Medical students and the electronic health record: 'an epic use of time'. Am J Med 2014;127(09): 891-895

5 Schenarts PJ, Schenarts KD. Educational impact of the electronic medical record. J Surg Educ 2012;69(01):105-112

6 Spencer DC, Choi D, English C, Girard D. The effects of electronic health record implementation on medical student educators. Teach Learn Med 2012;24(02):106-110

7 Hammoud MM, Dalymple JL, Christner JG, et al. Medical student documentation in electronic health records: a collaborative statement from the Alliance for Clinical Education. Teach Learn Med 2012;24(03):257-266

8 Association of American Medical C. Compliance Advisory. Electronic Health Records (EHRs) in Academic Health Centers; 2014. Available at: https://www.aamc.org/download/316610/data/advisory3achallengefortheelectronichealthrecordsofacademicinsti.pdf. Accessed October 8, 2018

9 Mintz M, Narvarte HJ, O’Brien KE, Papp KK, Thomas M, Durning SJ. Use of electronic medical records by physicians and students in academic internal medicine settings. Acad Med 2009;84(12): 1698-1704

10 Wittels K, Wallenstein J, Patwari R, Patel S. Medical student documentation in the electronic medical record: patterns of use and barriers. West J Emerg Med 2017;18(01):133-136

11 Solarte I, Könings KD. Discrepancies between perceptions of students and deans regarding the consequences of restricting students' use of electronic medical records on quality of medical education. BMC Med Educ 2017;17(01):55

12 Friedman E, Sainte M, Fallar R. Taking note of the perceived value and impact of medical student chart documentation on education and patient care. Acad Med 2010;85(09):1440-1444 
13 Lehmann CU; Council on Clinical Information Technology. Pediatric aspects of inpatient health information technology systems. Pediatrics 2015;135(03):e756-e768

14 Wald JS, Haque SN, Rizk S, et al. Enhancing health IT functionality for children: the 2015 children's EHR format. Pediatrics 2018;141 (04):e20163894

15 Krist AH, Beasley JW, Crosson JC, et al. Electronic health record functionality needed to better support primary care. J Am Med Inform Assoc 2014;21(05):764-771

16 Tierney WM, Overhage JM, McDonald CJ, Wolinsky FD. Medical students' and housestaff's opinions of computerized order-writing. Acad Med 1994;69(05):386-389

17 Stephens MB, Corcoran TS, Motsinger C. Clinical documentation in electronic medical records: the student perspective. Med Sci Educ 2011;21(01):3-6

18 Harle CA, Gruber LA, Dewar MA. Factors in medical student beliefs about electronic health record use. Perspect Health Inf Manag 2014; $11: 1 \mathrm{f}$

19 Rouf E, Chumley HS, Dobbie AE. Electronic health records in outpatient clinics: perspectives of third year medical students. BMC Med Educ 2008;8:13

20 Heiman HL, Rasminsky S, Bierman JA, et al. Medical students' observations, practices, and attitudes regarding electronic health record documentation. Teach Learn Med 2014;26(01): 49-55
21 Lehmann CU, O'Connor KG, Shorte VA, Johnson TD. Use of electronic health record systems by office-based pediatricians. Pediatrics 2015;135(01):e7-e15

22 Peled JU, Sagher O, Morrow JB, Dobbie AE. Do electronic health records help or hinder medical education? PLoS Med 2009;6(05): e1000069

23 Johnson KB, Lehmann CU; Council on Clinical Information Technology of the American Academy of Pediatrics. Electronic prescribing in pediatrics: toward safer and more effective medication management. Pediatrics 2013;131(04):e1350-e1356

24 Caceres JW, DiCorcia MJ. The impact of technology on the development of core entrustable professional activities (EPAs). Med Sci Educ 2017;8(01):247-249

25 Gagliardi JP, Turner DA. The electronic health record and education: rethinking optimization. J Grad Med Educ 2016;8(03): 325-327

26 Alkureishi MA, Lee WW, Lyons M, et al. Impact of electronic medical record use on the patient-doctor relationship and communication: a systematic review. J Gen Intern Med 2016;31(05): 548-560

27 Pereira AG, Kim M, Seywerd M, Nesbitt B, Pitt MB; Minnesota Epic101 Collaborative. Collaborating for competency-a model for single electronic health record onboarding for medical students rotating among separate health systems. Appl Clin Inform 2018;9 (01):199-204 